\title{
Starting Renal Replacement Therapy: Is It About Time?
}

\author{
Elaine $\mathrm{Ku}^{\mathrm{a}}{ }^{\mathrm{a}} \mathrm{b}$ Charles E. McCulloch ${ }^{c}$ Kirsten L. Johansen ${ }^{\mathrm{a}, \mathrm{c}}$ \\ ${ }^{a}$ Division of Nephrology, Department of Medicine, University of California, San Francisco, CA, USA; ${ }^{b}$ Division of \\ Pediatric Nephrology, Department of Pediatrics, University of California, San Francisco, CA, USA; ' Department of \\ Epidemiology and Biostatistics, University of California, San Francisco, CA, USA
}

\section{Keywords}

Chronic kidney disease - End-stage renal disease - Timing of dialysis initiation

\begin{abstract}
Background: Studies of the timing of end-stage renal disease (ESRD) have primarily defined "early" versus "late" initiation of dialysis using estimated glomerular filtration rate (eGFR)-based criteria. Our objective was to determine the theoretical time that could be spent in chronic kidney disease (CKD) stage 5 prior to reaching a conservative eGFR threshold of $5 \mathrm{~mL} / \mathrm{min} / 1.73 \mathrm{~m}^{2}$ compared to the actual time spent in CKD stage 5 by risk factors of interest. Methods: Eight-hundred and seventy Chronic Renal Insufficiency Cohort participants with CKD stage 5 who started renal replacement therapy (RRT) were included for retrospective study. We used mixed models to estimate the person-specific trajectory of renal function. We then used these individual trajectories to estimate the amount of time that would be spent in CKD stage 5 (between eGFR of 15 and $5 \mathrm{~mL} / \mathrm{min} / 1.73 \mathrm{~m}^{2}$ ) and compared this estimate to the actual time spent in CKD stage 5 prior to ESRD (between eGFR of $15 \mathrm{~mL} / \mathrm{min} / 1.73 \mathrm{~m}^{2}$ and ESRD). Results: We found the median observed time between eGFR of $15 \mathrm{~mL} / \mathrm{min} / 1.73 \mathrm{~m}^{2}$ to RRT was 9.6 months, but the median predicted time between eGFR of $15 \mathrm{~mL} /$ $\mathrm{min} / 1.73 \mathrm{~m}^{2}$ to eGFR of $5 \mathrm{~mL} / \mathrm{min} / 1.73 \mathrm{~m}^{2}$ was 17.7 months.
\end{abstract}

Some of the largest differences between the predicted and actual amount of time spent in CKD stage 5 were noted among those with systolic blood pressure <140 mm Hg (9.7 months longer predicted compared to actual), proteinuria $<1 \mathrm{~g} / \mathrm{g}$ (9.1 months), and serum albumin $\geq 3.5 \mathrm{~g} / \mathrm{dL}$ (9.0 months). Conclusion: We found marked differences between the actual and predicted time spent in CKD stage 5 based on risk factors of interest. We believe that placing timing of dialysis initiation in the perspective of time is novel and may identify subgroups of patients who may derive particular benefit from a more concerted effort to delay RRT.

(c) 2019 S. Karger AG, Basel

\section{Introduction}

The transition from advanced chronic kidney disease $(\mathrm{CKD})$ to end-stage renal disease (ESRD) represents a vulnerable period, when multiple physiologic and psychosocial changes occur as patients prepare for either dialysis or kidney transplantation. Observational studies have suggested a lack of survival benefit to early initiation of dialysis or earlier preemptive transplantation [14]. A large randomized controlled trial (the Initiating Dialysis Early and Late trial) also did not show a survival benefit to earlier (estimated glomerular filtration rate [eGFR] of $10-14 \mathrm{~mL} / \mathrm{min} / 1.73 \mathrm{~m}^{2}$ ) versus later dialysis

\section{KARGER}

(c) 2019 S. Karger AG, Basel

E-Mail karger@karger.com

www.karger.com/ajn
Elaine $\mathrm{Ku}, \mathrm{MD}$

Division of Nephrology, Department of Medicine

University of California, 521 Parnassus Avenue

C443, Box 0532, San Francisco, CA 94143-0532 (USA)

E-Mail elaine.ku@ucsf.edu 
initiation (5-7 mL/min/1.73 $\mathrm{m}^{2}$ ) [2]. The 2015 Kidney Disease Outcomes Quality Initiative guidelines for hemodialysis do not currently specify an eGFR threshold at which dialysis should be initiated [5]. However, in the United States, over half of all patients begin dialysis at an eGFR above $10 \mathrm{~mL} / \mathrm{min} / 1.73 \mathrm{~m}^{2}$ despite the lack of known benefit to earlier renal replacement therapy (RRT) initiation [6].

A number of studies have examined the timing of initiation of RRT based on the level of eGFR at the start of dialysis or receipt of kidney transplant [7-12]. However, few studies have characterized "early" versus "late" RRT initiation based on the amount of additional time that could be gained if nephrologists were to adopt a strategy of explicitly targeting a low eGFR threshold (e.g., eGFR of $5 \mathrm{~mL} / \mathrm{min} / 1.73 \mathrm{~m}^{2}$ ) in clinical practice. Quantifying "early" versus "late" RRT initiation in metrics of time as opposed to eGFR level may be more useful, given that the amount of time left before dialysis is of greatest concern for most patients with CKD stage 5. For providers, these data may provide a framework for discussions regarding the time that could potentially be available if patients were to partner with their providers to try to delay the initiation of dialysis for as long as possible.

In this study, our objective was to compare the predicted amount of time that would be spent between entry into CKD stage 5 and eGFR of $5 \mathrm{~mL} / \mathrm{min} / 1.73 \mathrm{~m}^{2}$ against the actual observed time between entry into CKD stage 5 and initiation of RRT among participants of the Chronic Renal Insufficiency Cohort (CRIC) study. We also examined the extent to which risk factors known to affect CKD progression were associated with differences between the predicted and actual time between CKD stage 5 and RRT initiation.

\section{Materials and Methods}

\section{Study Population}

The CRIC study is a NIH-sponsored, multicenter, observational cohort that enrolled patients from 7 clinical centers located throughout the United States [13]. Participants with eGFR between 20 and $70 \mathrm{~mL} / \mathrm{min} / 1.73 \mathrm{~m}^{2}$ based on the Modification of Diet in Renal Disease equation were recruited for study between June 2003 and September 2008. The inclusion and exclusion criteria have been previously published $[13,14]$. CRIC participants were followed annually at in-person study visits, during which medical history, medication use, comorbidity, and laboratory data were routinely assessed. For this study, we included the 870 CRIC participants with data available in CKD stage 5 (eGFR below $15 \mathrm{~mL} / \mathrm{min} / 1.73 \mathrm{~m}^{2}$ ), of whom 772 started RRT during our followup period.

Time in CKD Stage 5 to ESRD
Modeling Renal Function Trajectory across CKD Stages

We estimated renal function using the CKD-EPI creatininebased equation [15]. We used creatinine to estimate GFR because it is the biomarker most commonly used in clinical practice and therefore results can be most easily extrapolated to patient care. We used linear mixed modeling to estimate changes in eGFR over time from CRIC enrollment until ESRD onset using all data available from time of entry into CRIC. Our mixed models included person-specific linear and quadratic time terms to accommodate nonlinearities in eGFR trajectory and provide a flexible fit for each individual as previously described [16]. We adjusted these mixed models for age at the time of cohort entry and race (black vs. nonblack).

\section{Examination of Time Spent in Each CKD Stage According to}

Factors of Interest

We first categorized patients by their predicted versus actual observed time to RRT into 4 scenarios: (1) predicted time longer than actual observed time to RRT with RRT occurring during the follow-up period; (2) predicted time shorter than actual observed time to RRT with RRT occurring during the follow-up period (3) predicted time shorter than end of study with no observed RRT during the follow-up period, and (4) predicted time longer than end of study with no observed RRT during the follow-up period. The goal of this analysis was to provide an overall qualitative description of the distribution of patients included for analysis.

Next, we used the person-specific trajectories described above to estimate differences in the predicted versus actual amount of time spent in CKD stage 5 based on the presence or absence of individual factors of interest. For this analysis, we only included the 772 participants who were observed to develop ESRD during our follow-up period. Factors that we examined included demographic characteristics (age at entry into CKD stage 5 [categorized as $\geq 60$ vs. $<60$ years], sex, and race and ethnicity [white, black, or Hispanic]), comorbidities (presence or absence of cardiovascular disease [by self-report], uncontrolled blood pressure [systolic blood pressure (SBP) $\geq$ vs. $<140 \mathrm{~mm} \mathrm{Hg}$ ], obesity [body mass index $\geq$ or $<30 \mathrm{~kg} / \mathrm{m}^{2}$ ], diabetes [yes/no], tobacco use [yes/no], and medication use [angiotensin-converting enzyme (ACE) inhibitors or angiotensin receptor blockers (ARB), statins, and diuretics]), and laboratory findings that may factor into the decision to initiate RRT (hemoglobin A1c among diabetic patients [ $<$ or $\geq 7.5 \%$ ], proteinuria $[<$ or $\geq 1 \mathrm{~g} / \mathrm{g}$ ], serum albumin $[\geq$ or $<3.5 \mathrm{~g} / \mathrm{g}$ ], sodium $[\geq$ or $<135 \mathrm{meq} / \mathrm{L}$ ], potassium [ $\geq$ or $<5.0 \mathrm{meq} / \mathrm{L}$ ], bicarbonate [ $\geq$ or $<22 \mathrm{meq} / \mathrm{L}]$, and serum blood urea nitrogen $[\geq$ or $<50 \mathrm{mg} / \mathrm{dL}]$ ). For all nonfixed covariates, we used the values at the first visit upon entry into CKD stage 5 (eGFR of $15 \mathrm{~mL} / \mathrm{min} / 1.73 \mathrm{~m}^{2}$ ) to define their presence or absence as risk factors for progression.

We tested whether the differences between the predicted time to eGFR of $5 \mathrm{~mL} / \mathrm{min} / 1.73 \mathrm{~m}^{2}$ and the actual time to RRT based on each factor of interest were statistically significantly different using chi-square, Wilcoxon rank sum, or Kruskal-Wallis tests. We chose an eGFR threshold of $5 \mathrm{~mL} / \mathrm{min} / 1.73 \mathrm{~m}^{2}$ to define "late" RRT initiation since much of the literature on the timing of dialysis initiation has used this as the lower eGFR threshold of interest, and this was the lower threshold in one of the treatment groups in the Initiating Dialysis Early and Late trial [2].

Although our main focus was on unadjusted analyses as our primary models, we also used multivariate logistic regression including all demographic, comorbidity, and laboratory data as pre- 
dictors of interest to determine which factors were most strongly associated with odds of a $>8$-month difference between the predicted and actual time spent in CKD stage 5 among those observed to develop ESRD during our follow-up period. We selected an 8 -month period as our outcome of interest because this represented the median difference between the predicted and actual time spent in CKD stage 5. We used backward selection (with a threshold $p$ value of $<0.10$ ) to identify the factors most predictive of early RRT initiation and included these factors in our final multivariate logistic regression model.

We used deidentified data from the National Institute of Diabetes and Digestive and Kidney Diseases (NIDDK) Biorepository for analysis, which administratively censored data as of March 2013. The UCSF Institutional Review Board considers this study exempt human subjects research.

\section{Results}

\section{Study Participants}

Characteristics of participants included for analysis at the first visit when eGFR fell below $15 \mathrm{~mL} /$ $\mathrm{min} / 1.73 \mathrm{~m}^{2}$ are shown in Table 1 . Median age was 60.8 years, $52 \%$ were black, and $66 \%$ had diabetes. Mean hemoglobin was $11.4 \mathrm{~g} / \mathrm{dL}$, and median proteinuria was $1.7 \mathrm{~g} / \mathrm{g}$. Participants who were excluded were older and had higher BUN and lower proteinuria. Of the 870 participants who entered CKD stage 5, 754 (87\%) had predicted time to ESRD that was longer than the actual observed time to ESRD, versus only 18 participants (2\%) had predicted time to ESRD that was shorter than the actual observed time to ESRD. In addition, among those not observed to develop ESRD during the followup period (98 out of 870 participants), 6 participants had a predicted time to ESRD that was shorter than the end of study, and 92 participants had a predicted time to ESRD that would have been longer than the end of study.

\section{Factors Associated with Observed Time to RRT}

Characteristics of participants who entered CKD stage 5 but did not initiate RRT during our study period are shown in online supplementary Table 1, see www.karger. com/doi/10.1159/000501510. Among the 98 participants who did not develop ESRD, 51 died during the study follow-up period. The observed median time to RRT was 9.6 months, but the median predicted time to an eGFR of $5 \mathrm{~mL} / \mathrm{min} / 1.73 \mathrm{~m}^{2}$ was 17.7 months. Thus, the overall median difference between predicted and actual time spent in CKD stage 5 was 8.1 months.

We examined the amount of observed (i.e., actual) time spent in CKD stage 5 according to each factor of in-
Table 1. Baseline characteristics of CRIC participants at entry into CKD stage 5

\begin{tabular}{lc}
\hline Baseline characteristics $(n=870)$ & $n(\%)$ or mean \pm SD \\
\hline Age, years, median (IQR) & $60.9(52.2-67.8)$ \\
Gender, female & $380(43.7)$ \\
Race/ethnicity & $213(24.5)$ \\
$\quad$ White & $453(52.1)$ \\
$\quad$ Black & $166(19.1)$ \\
$\quad$ Hispanic & $38(4.4)$ \\
Other & $496(57.1)$ \\
Obese & $393(45.2)$ \\
Any cardiovascular disease & $573(65.9)$ \\
Diabetes & $137.3(122.7-155.3)$ \\
SBP, mm Hg, median (IQR) & \\
Attributed cause of renal disease & $367(42)$ \\
$\quad$ Diabetes & $171(20)$ \\
$\quad$ Hypertension & $84(10)$ \\
Other & $248(29)$ \\
$\quad$ Unknown & $137(15.8)$ \\
Current smoker & $4.5 \pm 0.6$ \\
Serum potassium, mEq/L & $11.4 \pm 1.6$ \\
Hemoglobin, g/dL & $22.1 \pm 3.5$ \\
Serum CO, mmol/L & $50.8 \pm 18.0$ \\
Serum urea nitrogen, mg/dL & $3.7 \pm 0.5$ \\
Serum albumin, g/dL & \\
Urine protein/creatinine ratio, & $1.7(0.7-3.8)$ \\
$\quad$ g/g, median (IQR) & \\
\hline
\end{tabular}

CRIC, Chronic Renal Insufficiency Cohort; IQR, interquartile range; SBP, systolic blood pressure.

terest in separate univariate analyses. We found that demographic and comorbid factors including age, race, presence of CVD, and smoking were not statistically significantly associated with the observed time spent in CKD stage 5 (Fig. 1a, see blue bars). In contrast, female sex, obesity, lack of diabetes, controlled SBP, and proteinuria $<1 \mathrm{~g} / \mathrm{g}$ were associated with statistically significantly longer observed time in CKD stage 5 (Fig. 1a, see blue bars). Low sodium, lower serum urea nitrogen, lower A1c, and lower serum albumin were associated with shorter time spent in CKD stage 5 (Fig. 1b). In contrast, use of various medications including ACE/ARBs, statins, and diuretics was not associated with time spent in CKD stage 5 .

\section{Differences between Time to RRT and Predicted Time} to eGFR of $5 \mathrm{~mL} / \mathrm{min} / 1.73 \mathrm{~m}^{2}$

Next, we determined the additional time that could have been spent in CKD stage 5 if an eGFR threshold of $5 \mathrm{~mL} / \mathrm{min} / 1.73 \mathrm{~m}^{2}$ had been used for RRT initiation according to each factor of interest in univariate analysis. 


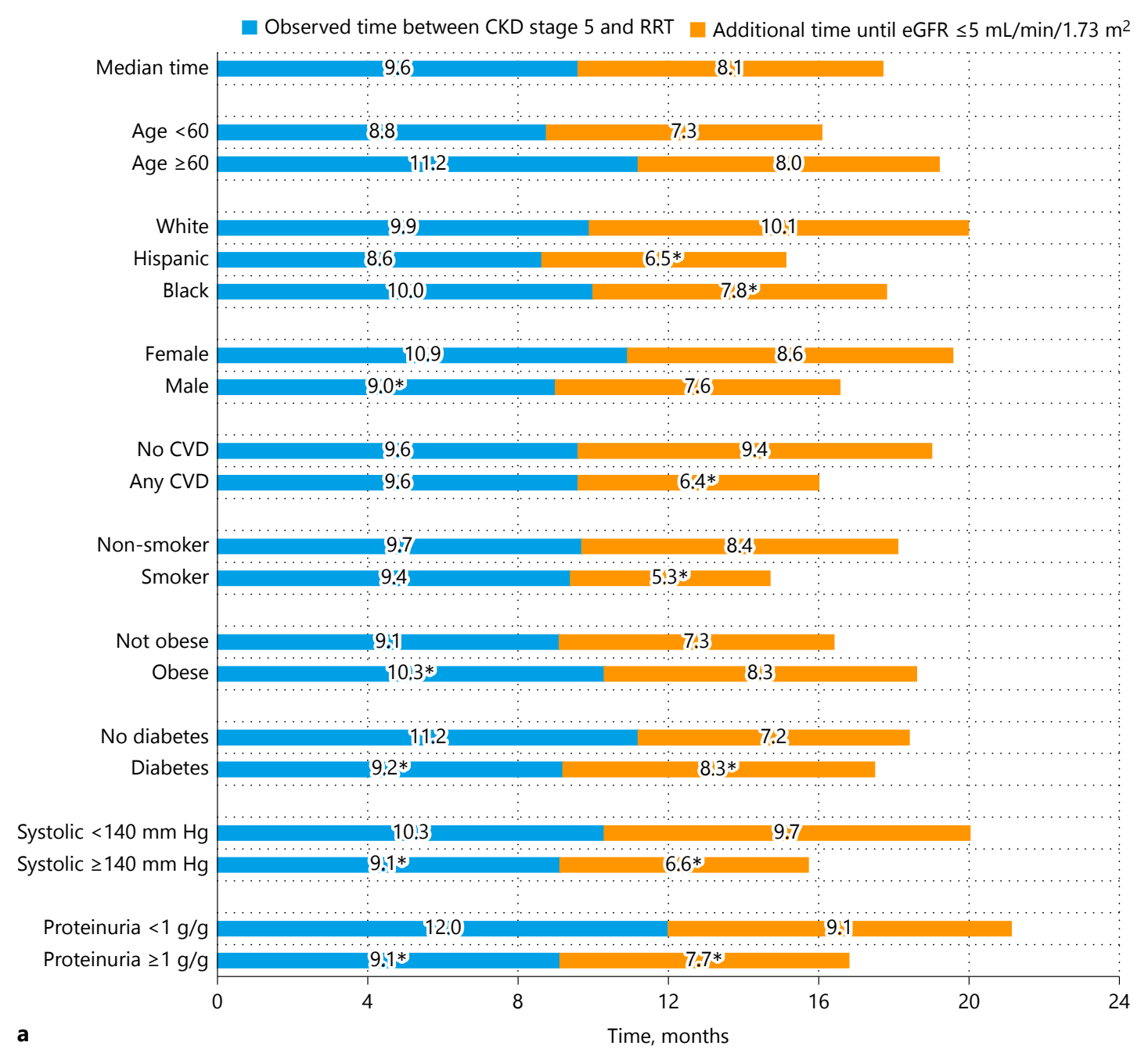

Fig. 1. a Observed versus predicted time available in CKD stage 5 based on demographic and comorbid data associated with progression of CKD. Reference group used in tests for statistically significant differences for all characteristics is the first row for each subdivision (e.g., age $<60$ years, white race). b Observed versus predicted time available in CKD stage 5 based on medication and

The amount of additional time was longest for white participants (10.1 months), participants with no cardiovascular disease (9.4 months), low proteinuria (9.1 months), low serum urea nitrogen concentration (10.1 months), and high serum albumin concentration (9.0 months) as shown in Figure 1b (orange bars). In contrast, the smallest differences between the predicted and actual time spent in CKD stage 5 were observed among participants with low serum sodium (1.6 months, Fig. 1a, orange bars) and among smokers (5.3 months, Fig. 1b). laboratory data associated with progression of CKD. Reference group used in tests for statistically significant differences for all characteristics is the first row for each subdivision (e.g., no diuretic, no ACE/ARB). CKD, chronic kidney disease; RRT, renal replacement therapy; eGFR, estimated glomerular filtration rate; SBP, systolic blood pressure. ${ }^{*} p<0.05$ compared to the reference group.

(Figure continued on next page.)

When we used multivariate logistic regression models to determine factors independently associated with larger differences between the predicted and actual time to initiation of RRT (greater than the median), we found that age, race/ethnicity, cardiovascular disease, smoking status, SBP, and proteinuria were statistically significantly predictors (Table 2). In our final multivariate model, age $\geq 60$ years was associated with a 1.5 times higher odds of having a large difference between predicted and actual time to RRT (95\% CI 1.09-2.06). White race, nonsmok- 


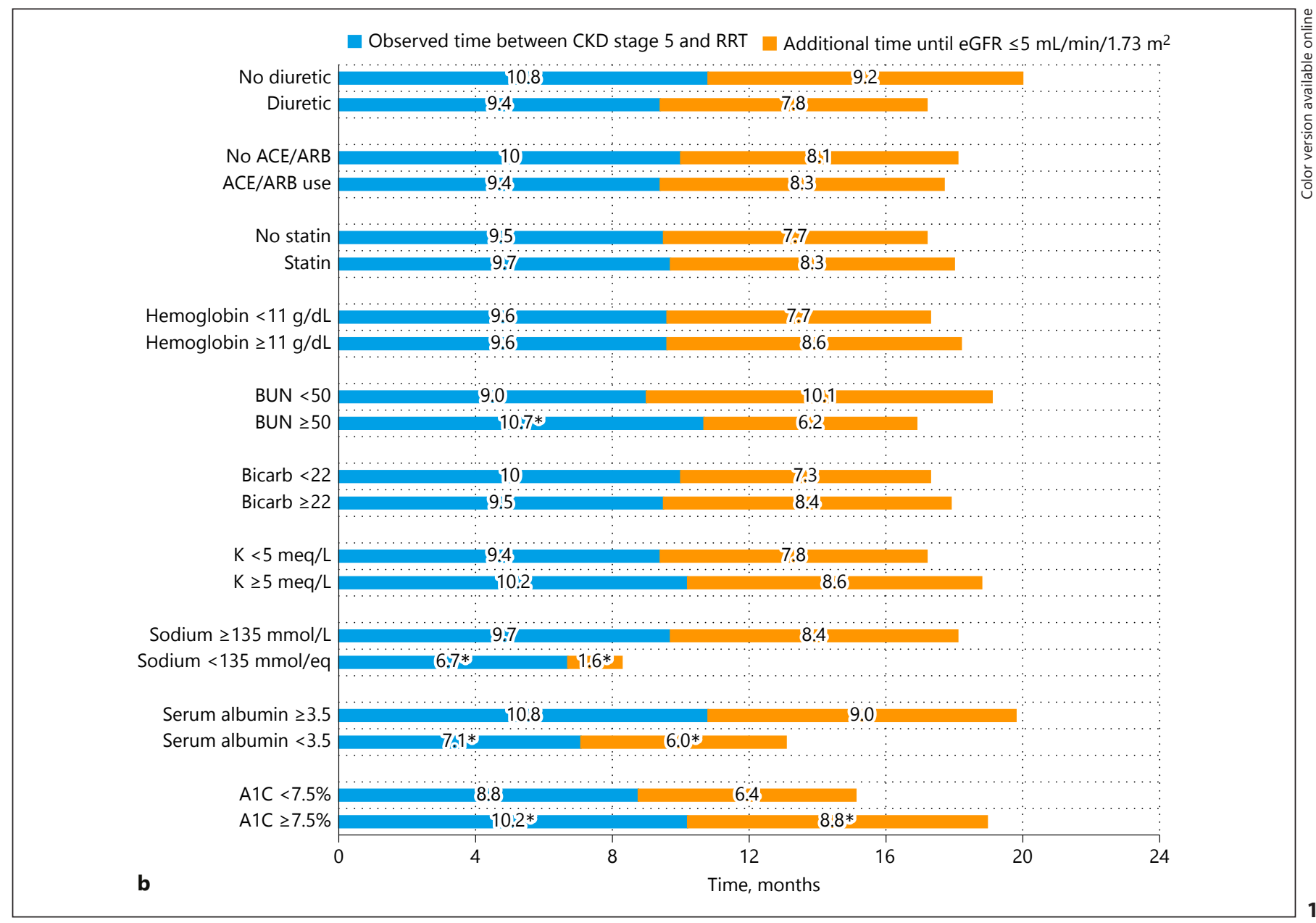

Table 2. Odds of starting dialysis earlier than expected ( $>8$ months prior to predicted time to eGFR of $<5 \mathrm{~mL} / \mathrm{min} / 1.73 \mathrm{~m}^{2}$ ) in final multivariate analysis

\begin{tabular}{ll}
\hline Characteristics $(n=772)$ & OR $(95 \% \mathrm{CI})$ \\
\hline $\begin{array}{l}\text { Age, years, } \geq 60 \text { vs. }<60 \\
\text { Race }\end{array} \quad 1.50(1.09-2.06)$ \\
$\quad$ White & $1.64(1.14-2.36)$ \\
$\quad$ Black & Ref. \\
$\quad$ Hispanic & $0.82(0.53-1.26)$ \\
No cardiovascular disease (versus & $1.75(1.28-2.41)$ \\
$\quad$ presence of CVD) & $1.59(1.03-2.45)$ \\
Non-smoker $($ versus smoker $)$ & $1.40(1.02-1.93)$ \\
SBP $<140 \mathrm{~mm}$ Hg (versus $\geq 140 \mathrm{~mm} \mathrm{Hg})$ & \\
Urine protein/creatinine ratio $<1 \mathrm{~g} / \mathrm{g}$ & $1.24(0.88-1.73)$ \\
$\quad($ versus $\geq 1 \mathrm{~g} / \mathrm{g})$ &
\end{tabular}

eGFR, estimated glomerular filtration rate; SBP, systolic blood pressure. ers, absence of cardiovascular disease, and controlled SBP were also associated with higher odds of larger discrepancies between the predicted and actual time available in CKD stage 5 prior to RRT (Table 2).

\section{Discussion}

Most observational and randomized controlled studies have not demonstrated a benefit to early initiation of RRT, and some studies have suggested that this strategy may be associated with harm [17-19]. Yet, despite these data, the mean eGFR at the time of dialysis initiation has increased to over $10 \mathrm{~mL} / \mathrm{min} / 1.73 \mathrm{~m}^{2}$ over the last decade $[6,20]$. Although many studies have examined reasons for early versus late RRT initiation based on eGFR criteria, few studies have been able to quantify the magnitude of additional time that could be spent in CKD stage 5 if RRT were to be initiated at a more conservative eGFR 
threshold (e.g., $<5 \mathrm{~mL} / \mathrm{min} / 1.73 \mathrm{~m}^{2}$ ). In our study, we found that even among CRIC participants, RRT could potentially be delayed by a median of 8 months if patients could be managed medically until reaching an eGFR of 5 $\mathrm{mL} / \mathrm{min} / 1.73 \mathrm{~m}^{2}$. The greatest discrepancies between the actual and predicted times spent in CKD stage 5 were noted among white participants, those with controlled SBP and without substantial proteinuria, and in the absence of cardiovascular disease in both univariate and multivariate analyses. In contrast, tobacco use, low serum sodium, and higher serum urea nitrogen were associated with smaller discrepancies between the observed and predicted time in CKD stage 5 . We believe our study identifies characteristics of individuals who may benefit from a more concerted effort to delay the initiation of RRT.

Currently, the Kidney Disease Outcomes Quality Initiative guidelines recommend that the decision to initiate dialysis should not be based solely on any particular eGFR threshold among asymptomatic patients [5]. However, current trends in practice still show that dialysis is being initiated at higher eGFR levels despite the lack of demonstrated survival or quality of life benefit with this approach $[9,20]$. We believe our estimation of the potential magnitude of additional time that could be spent in CKD stage 5 if RRT were started at a more conservative threshold is informative and supports the need to reassess the threshold for uremic symptoms that may warrant initiation of RRT. These data may be helpful to providers in timing the appropriate placement of dialysis access, counseling of patients regarding their prognosis, and identifying the subsets of patients in whom it may be possible to delay RRT initiation for a substantial period of time. Given that the average annual cost of hemodialysis is around USD 90,000 per year, delaying dialysis by 8 months could represent average savings of USD 60,000 per patient in the United States [21]. While we acknowledge that widespread implementation of a policy where dialysis is initiated later could provide cost-savings, such a treatment strategy will also necessitate more personnel support to closely monitor patients during the advanced stages of CKD if dialysis initiation is delayed.

Whereas prior randomized controlled trials have suggested a benefit to continuation of ACE inhibitors in the setting of CKD stage 4 [22], fewer studies have examined this question within a large number of patients with CKD stage 5. We found that continuation of ACE inhibitor or $\mathrm{ARB}$ use in CKD stage 5 was not associated with substantial differences in the observed time spent in CKD stage 5. The presence of uncontrolled proteinuria was associated with less additional predicted time in CKD stage 5, although this difference was small (1.4 months). It is possible that the relatively small estimated difference between predicted and observed time in stage 5 CKD based on proteinuria explains the lack of apparent benefit of ACE or ARB use, although we caution that our results are observational and may not imply causation.

Of interest, we did find older age to be associated with longer observed time spent in CKD stage 5, but the actual difference between those older versus younger than 60 years of age was small (on the order of 3 months). Although our findings are consistent with those of prior studies, which have described slower progression of CKD and later onset of RRT needs in older individuals [23,24], our data suggest that age may be less important than previously described when considered in terms of time. This discrepancy highlights the novelty of our study in its translation of risk estimates (represented by odds ratios) with associated time differences in the onset of RRT by risk factors such as age. There was also little difference in the additional time that could be available in CKD stage 5 by age based on the modeled time in CKD stage 5 .

Of the laboratory factors we studied, low serum urea nitrogen, high serum albumin, and eunatremia were associated with longer amount of additional time that could theoretically be spent in CKD stage 5 . However, none of these factors remained important in analysis that adjusted for other clinical factors. Surprisingly, elevated hemoglobin A1c was associated with longer observed time and larger discrepancies in the amount of additional time that could be spent in CKD stage 5. Although the reasons for this observation are unclear, we speculate that lower hemoglobin A1c among those with diabetes may reflect the severity of renal disease (i.e., "burnt out diabetes") rather than tight blood glucose control [25]. This possibility is supported by the observation that when we examined diabetes as the factor of interest (rather than hemoglobin A1c), those with diabetes spent less time in CKD stage 5 compared with those without diabetes (but the difference in time was small).

The strengths of our study include the use of a wellcharacterized, nationally representative cohort of CKD patients with longitudinal follow-up and careful ascertainment of comorbidities and outcomes of interest in one of the largest cohorts of patients with stage 5 CKD. In addition, we believe our time-centric approach to the study of timing of dialysis initiation to be novel and our data informative for providers and patients, especially for the purposes of prognostication and counseling. However, we note several limitations to our study, including its observational nature and the lack of granular data on 
acute kidney injury events that may have occurred in the immediate period prior to RRT initiation. We also do not have data on factors that influenced the actual decision to initiate RRT or the contribution of symptoms that may have driven such decisions. In addition, participants in CRIC Study may not be representative of nonstudy participants, and the amount of time spent in CKD that we describe may be representative of participants receiving optimal therapy under the care of nephrologists. However, we do note that a prior study using the CRIC cohort has described the median eGFR at time of dialysis initiation to be around $10 \mathrm{~mL} / \mathrm{min} / 1.73 \mathrm{~m}^{2}$, which is consistent with practice patterns in the United States as described previously [26]. Finally, we acknowledge that our findings may be reflective of practice patterns in the United States and may not reflect practice patterns in other countries.

In conclusion, we believe that initiation of RRT occurs substantially earlier than would be predicted, although we are unable to pinpoint the exact reasons for the decision to start dialysis. Aside from eGFR, there appear to other factors associated with the time spent in CKD stage 5 and the decision to initiate dialysis. We have identified characteristics of individuals who may be more likely to have slow progression of disease and spend a significant amount of time in CKD stage 5. Further studies are needed to identify the subset of patients who may safely delay initiation of RRT and to elucidate the impetus behind the complex decision to initiate RRT by both patients and providers alike.

\section{Acknowledgements}

This work was supported by the National Institutes of Health R01 DK115629 to EK and KLJ and K24 DK085153 to KLJ. The CRIC Study was conducted by the CRIC Study Investigators and supported by the NIDDK. The data from the CRIC Study reported here were supplied by the NIDDK Central Repositories. This manuscript was not prepared in collaboration with Investigators of the CRIC Study and does not necessarily reflect the opinions or views of the CRIC Study, the NIDDK Central Repositories, or the NIDDK.

\section{Disclosure Statement}

The authors have no conflict of interest to declare.

\section{References}

1 Akkina SK, Connaire JJ, Snyder JJ, Matas AJ, Kasiske BL. Earlier is not necessarily better in preemptive kidney transplantation. Am J Transplant. 2008 Oct;8(10):2071-6.

2 Cooper BA, Branley P, Bulfone L, Collins JF, Craig JC, Fraenkel MB, et al.; IDEAL Study. A randomized, controlled trial of early versus late initiation of dialysis. N Engl J Med. 2010 Aug;363(7):609-19.

3 Korevaar JC, Jansen MA, Dekker FW, Boeschoten EW, Bossuyt PM, Krediet RT; NECOSAD Study Group. Evaluation of DOQI guidelines: early start of dialysis treatment is not associated with better health-related quality of life. Am J Kidney Dis. 2002 Jan;39(1):108-15.

4 Pan Y, Xu XD, Guo LL, Cai LL, Jin HM. Association of early versus late initiation of dialysis with mortality: systematic review and meta-analysis. Nephron Clin Pract. 2012; 120(3):c121-31.

5 Daugirdas JT, Depner TA, Inrig J, Mehrotra R, Rocco MV, Suri RS, et al.; National Kidney Foundation. 2015 update. Am J Kidney Dis. 2015 Nov;66(5):884-930.

6 Leurs P, Machowska A, Lindholm B. Timing of dialysis initiation: when to start? Which treatment? J Ren Nutr. 2015 Mar;25(2):238-41.

7 Bargman JM. Timing of Initiation of RRT and Modality Selection. Clin J Am Soc Nephrol. 2015 Jun;10(6):1072-7.
8 Crews DC, Scialla JJ, Boulware LE, Navaneethan SD, Nally JV Jr, Liu X, et al.; DEcIDE Network Patient Outcomes in End Stage Renal Disease Study Investigators. Comparative effectiveness of early versus conventional timing of dialysis initiation in advanced CKD. Am J Kidney Dis. 2014 May;63(5):806-15.

9 O'Hare AM, Wong SP, Yu MK, Wynar B, Perkins M, Liu CF, et al. Trends in the Timing and Clinical Context of Maintenance Dialysis Initiation. J Am Soc Nephrol. 2015 Aug;26(8): 1975-81.

10 Slinin Y, Guo H, Li S, Liu J, Morgan B, Ensrud $\mathrm{K}$, et al. Provider and care characteristics associated with timing of dialysis initiation. Clin J Am Soc Nephrol. 2014 Feb;9(2):310-7.

11 Yu MK, O’Hare AM, Batten A, Sulc CA, Neely EL, Liu CF, et al. Trends in Timing of Dialysis Initiation within Versus Outside the Department of Veterans Affairs. Clin J Am Soc Nephrol. 2015 Aug;10(8):1418-27.

12 Rosansky SJ. Early dialysis initiation, a look from the rearview mirror to what's ahead. Clin J Am Soc Nephrol. 2014 Feb;9(2):222-4.

13 Feldman HI, Appel LJ, Chertow GM, Cifelli D, Cizman B, Daugirdas J, et al.; Chronic Renal Insufficiency Cohort (CRIC) Study Investigators. The Chronic Renal Insufficiency Cohort (CRIC) Study: design and Methods. J Am Soc Nephrol. 2003 Jul;14(7 Suppl 2): S148-53.
14 Lash JP, Go AS, Appel LJ, He J, Ojo A, Rahman M, et al.; Chronic Renal Insufficiency Cohort (CRIC) Study Group. Chronic Renal Insufficiency Cohort (CRIC) Study: baseline characteristics and associations with kidney function. Clin J Am Soc Nephrol. 2009 Aug; 4(8):1302-11.

15 Inker LA, Schmid CH, Tighiouart H, Eckfeldt JH, Feldman HI, Greene T, et al.; CKD-EPI Investigators. Estimating glomerular filtration rate from serum creatinine and cystatin C. N Engl J Med. 2012 Jul;367(1):20-9.

$16 \mathrm{Ku} \mathrm{E}$, Johansen KL, McCulloch CE. TimeCentered Approach to Understanding Risk Factors for the Progression of CKD. Clin J Am Soc Nephrol. 2018 May;13(5):693-701.

17 Beddhu S, Samore MH, Roberts MS, Stoddard GJ, Ramkumar N, Pappas LM, et al. Impact of timing of initiation of dialysis on mortality. J Am Soc Nephrol. 2003 Sep;14(9): 2305-12.

18 Kazmi WH, Gilbertson DT, Obrador GT, Guo H, Pereira BJ, Collins AJ, et al. Effect of comorbidity on the increased mortality associated with early initiation of dialysis. Am J Kidney Dis. 2005 Nov;46(5):887-96.

19 Susantitaphong P, Altamimi S, Ashkar M, Balk EM, Stel VS, Wright S, et al. GFR at initiation of dialysis and mortality in CKD: a meta-analysis. Am J Kidney Dis. 2012 Jun;59(6): 829-40. 
$20 \mathrm{Ku} \mathrm{E}$, Johansen KL, Portale AA, Grimes B, Hsu CY. State level variations in nephrology workforce and timing and incidence of dialysis in the United States among children and adults: a retrospective cohort study. BMC Nephrol. 2015 Jan;16(1):2.

21 United States Renal Data System: Annual Data Report. Chapter 9: Healthcare Expenditures for Persons with ESRD. Ntaional Institutes of Health, National Institute of Diabetes and Digestive and Kidney Diseases; 2018.
22 Hou FF, Zhang X, Zhang GH, Xie D, Chen PY, Zhang WR, et al. Efficacy and safety of benazepril for advanced chronic renal insufficiency. N Engl J Med. 2006 Jan;354(2):131-40.

23 Bao Y, Dalrymple L, Chertow GM, Kaysen GA, Johansen KL. Frailty, dialysis initiation, and mortality in end-stage renal disease. Arch Intern Med. 2012 Jul;172(14):1071-7.

24 Crews DC, Scialla JJ, Liu J, Guo H, BandeenRoche K, Ephraim PL, et al.; Developing Evidence to Inform Decisions about Effectiveness (DEcIDE) Patient Outcomes in End
Stage Renal Disease Study Investigators. Predialysis health, dialysis timing, and outcomes among older United States adults. J Am Soc Nephrol. 2014 Feb;25(2):370-9.

25 Kovesdy CP, Park JC, Kalantar-Zadeh K. Glycemic control and burnt-out diabetes in ESRD. Semin Dial. 2010 Mar-Apr;23(2):14856.

26 Rosansky SJ, Clark WF, Eggers P, Glassock RJ. Initiation of dialysis at higher GFRs: is the apparent rising tide of early dialysis harmful or helpful? Kidney Int. 2009 Aug;76(3):257-61. 